\title{
Pulse Interval Modulation for Ultra-High Speed IR-UWB Communications Systems
}

\author{
Marijan Herceg, Tomislav Švedek, and Tomislav Matić \\ Department of Communication, Faculty of Electrical Engineering, J.J.Strossmayer University of Osijek, \\ Kneza Trpimira 2b, 31000 Osijek, Croatia \\ Correspondence should be addressed to Marijan Herceg, marijan.herceg@etfos.hr
}

Received 16 February 2010; Revised 6 May 2010; Accepted 21 July 2010

Academic Editor: Jacques Palicot

Copyright (C) 2010 Marijan Herceg et al. This is an open access article distributed under the Creative Commons Attribution License, which permits unrestricted use, distribution, and reproduction in any medium, provided the original work is properly cited.

This paper analyzes performances of the Pulse Interval Modulation (PIM) scheme for impulse radio ultra-wideband (IR-UWB) communication systems. Due to the PIM anisochronous nature, a tap delay line (TDL) coded division multiple access (CDMA) scheme based on strict optical orthogonal codes (SOOC) is proposed. This scheme is suitable for multiuser high-speed data asynchronous transmission applications because the average symbol length is shorter than in Pulse Position Modulation (PPM) schemes and it needs only chip synchronization. The error probability over the additive white Gaussian noise (AWGN) channel is derived in the single- and multi-user environment and compared with other modulation schemes.

\section{Introduction}

Trends in modern communication systems place high demands on low power consumption, high-speed transmission, and anti-interference characteristics. Therefore, impulse radio ultra-wideband (IR-UWB) [1] systems have recently gained increased popularity. Since IR-UWB symbols are transmitted by short pulses $(<2 \mathrm{~ns})$, energy has spread over the frequency bands of up to $10 \mathrm{GHz}$. These pulses have to follow strict regulations concerning power and spectrum restrictions defined by local authorities, like the Federal Communications Commission (FCC) [2] in the USA. Because of power and spectral properties of the transmitted IR-UWB pulses, different types of orthogonal pulse shapes are used to provide a higher spectral efficiency [3,4]. Derivation of the Gaussian pulse and modified Hermite pulses (MHPs), usually called Hermites [5], provides a wide range of various pulse combinations for IR-UWB transmission and for that reason they are most commonly used as pulse shapes. The state of art in IR-UWB systems is presented by many applicable modulation techniques like Pulse Amplitude Modulation (PAM), PPM, Pulse Shape Modulation (PSM), on-off-keying (OOK), and biphase modulation (BPM). A combination of the exposed modulation techniques (hybrid techniques) can provide system improvements in terms of the error probability, a higher data rate, a less complex receiver, or less power consumption. Many hybrid techniques for IRUWB communication systems have been applied recently, such as Pulse Position Amplitude Modulation (PPAM) [6], Biorthogonal Pulse Position Modulation (BPPM) [7], OOK-PSM [8], PPM-PSM [9], and hybrid Shape-Amplitude Modulation [10, 11]. Regarding pulse amplitudes, positions, and shapes, three types of modulations can be distinguished. In PAM and OOK modulation information is contained in the amplitude of the signal, PPM uses the position of the pulse to convey information, whereas in PSM, information is conveyed in the shape of the pulse.

PIM was first introduced in [12] for wireless optical communication systems. It is interesting because it displays a higher transmission capacity by eliminating unused time chips within each symbol and does not require both chip and symbol synchronization, but only chip synchronization, since each symbol is initiated with a pulse. Different anisochronous and synchronous pulse time modulation (PTM) techniques for optical short-range wireless communications are compared in [13]. 
TABLE 1: Mappings between source bits and transmitted chips for 4-PPM and 4-PIM (Example).

\begin{tabular}{lcc}
\hline Source bits & 4-PPM chips & 4-PIM chips \\
\hline 00 & 1000 & $1(0)$ \\
01 & 0100 & $1(0) 0$ \\
10 & 0010 & $1(0) 00$ \\
11 & 0001 & $1(0) 000$ \\
\hline
\end{tabular}

This paper is organized as follows. Section 2 describes basic properties of the PIM scheme. In Section 3, the proposed system model is described, while in Section 4 error performance analysis is made over the AWGN channel. Section 5 gives simulation results while some conclusions are given in Section 6.

\section{PIM Scheme Basics}

PIM is part of anisochronous PTM techniques. The main characteristic of anisochronous schemes is that they do not have a fixed symbol structure, which means that the symbol length varies and is determined by the information content of the symbol. In PPM, each symbol has a fixed length and the chips following the pulse are redundant, while in PIM that redundancy is removed. A PIM symbol which encodes $M=\log _{2} L$ input bits, where $L$ is the number of symbols, is represented by a constant power pulse in the "ON" chip followed by the $(L-1)$ "OFF" chips. In order to avoid symbols in which the time between adjacent pulses is zero, an additional guard chip may also be added to each symbol immediately following the pulse. Table 1 shows the transformation of the source bit sequence into PPM chip sequences and PIM chip sequences with the guard zero chip.

The overall transmitted signal in the single-user case can be defined as

$$
s(t)=\sum_{m=0}^{\infty} \sqrt{E_{g}} p\left[t-T_{c}\left(2 m+\sum_{l=-\infty}^{m-1} S_{l}\right)\right],
$$

where $E_{g}$ is energy of the pulse, $T_{c}$ is chip duration, $p(t)$ is the pulse shape function (unit energy Gaussian monocycle with duration $T_{c}$ ), and $S_{l}$ is a random data sequence representing PIM coded data. If the maximum PIM symbol duration is limited to the PPM symbol duration, then the average symbol length of PIM with the guard zero chip is $L_{\mathrm{avg}}=$ $(L+3) / 2$. The achievable data rates for PIM and PPM are then

$$
\begin{aligned}
& R_{b-\mathrm{PIM}}=\frac{\log _{2} L}{L_{\mathrm{avg}} T_{c}}, \\
& R_{b-\mathrm{PPM}}=\frac{\log _{2} L}{L T_{c}},
\end{aligned}
$$

respectively. From (2) and (3), it can be clearly seen that the PIM bit rate is higher than PPM bit rate, due to the shorter average symbol length $L_{\text {avg. }}$ On the other hand, due to the shorter symbol length, PIM encoded data sequence has the higher average power than the data sequence encoded by

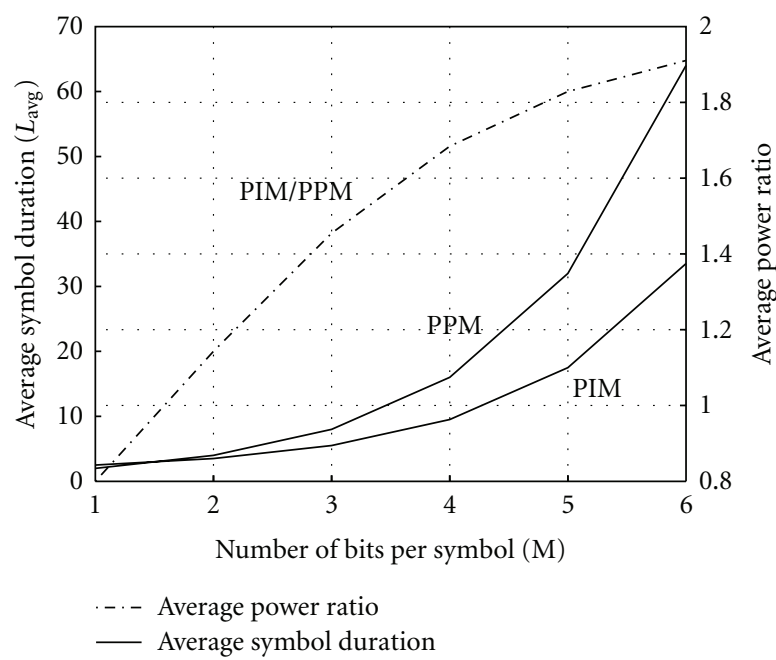

FIGURE 1: Dependence of symbol duration $\left(L_{\text {avg }}\right)$ and average power ratio (Pow PIM $/$ Pow $_{\text {PPM }}$ ) on the number of bits per symbol.

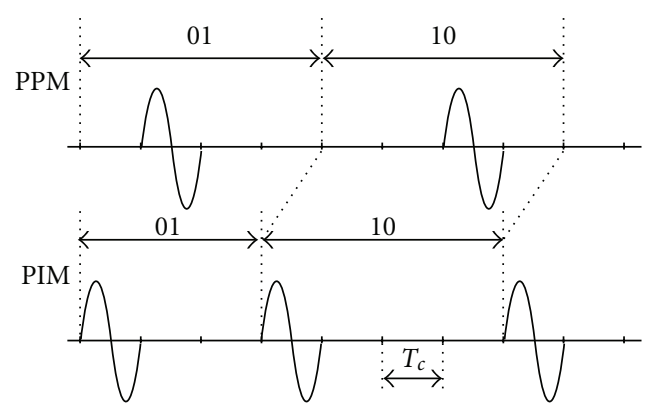

Figure 2: PPM and PIM symbol structure for the sequence of source bit combinations 01 and 10 .

using PPM. The average power per symbol for PIM and $\mathrm{PPM}$ is given as

$$
\begin{aligned}
& \text { PowpIM }=\frac{1}{L_{\mathrm{avg}} T_{c}} \int_{-\infty}^{\infty} p^{2}(t) d t, \\
& \text { PowPPM }=\frac{1}{L T_{c}} \int_{-\infty}^{\infty} p^{2}(t) d t,
\end{aligned}
$$

respectively. The dependence of the symbol length and the average power ratio (Pow PIM $_{\text {Pow }}$ PPM ) on the number of bits per symbol is shown in Figure 1.

Figure 2 shows an example of PPM and PIM symbols for the sequence of source bit combinations 01 and 10 .

\section{The Proposed System Model}

In this section, the performance of PIM in a multi-user environment is derived for IR-UWB communication systems. Due to the PIM anisochronous nature, CDMA based on the TDL is proposed in [14]. This scheme is suitable for IR-UWB systems because it needs only chip synchronization, while the time-hopping systems proposed in [6-11] need both frame and chip synchronization which increase hardware 


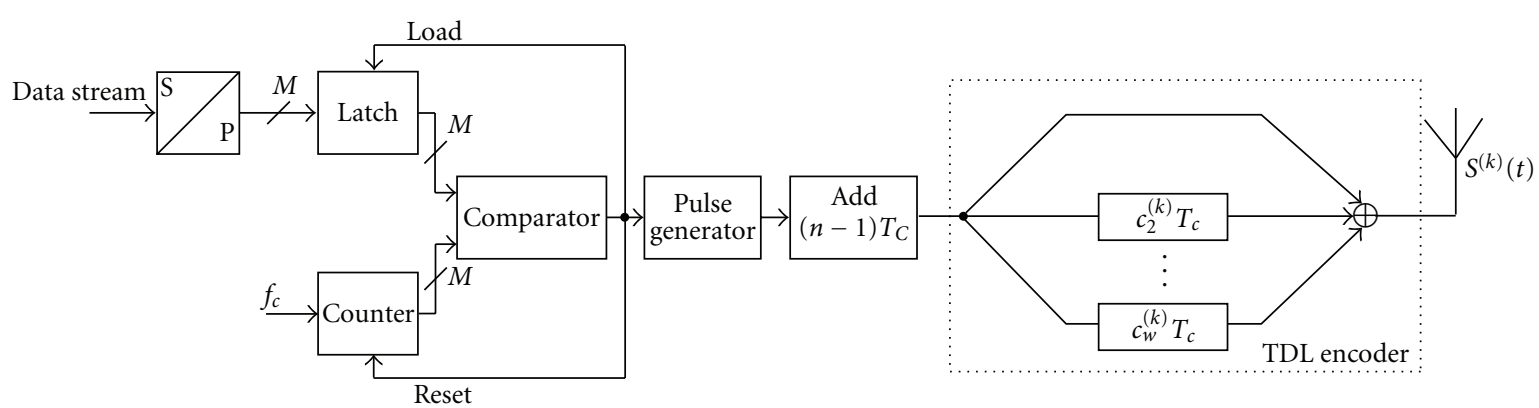

(a)

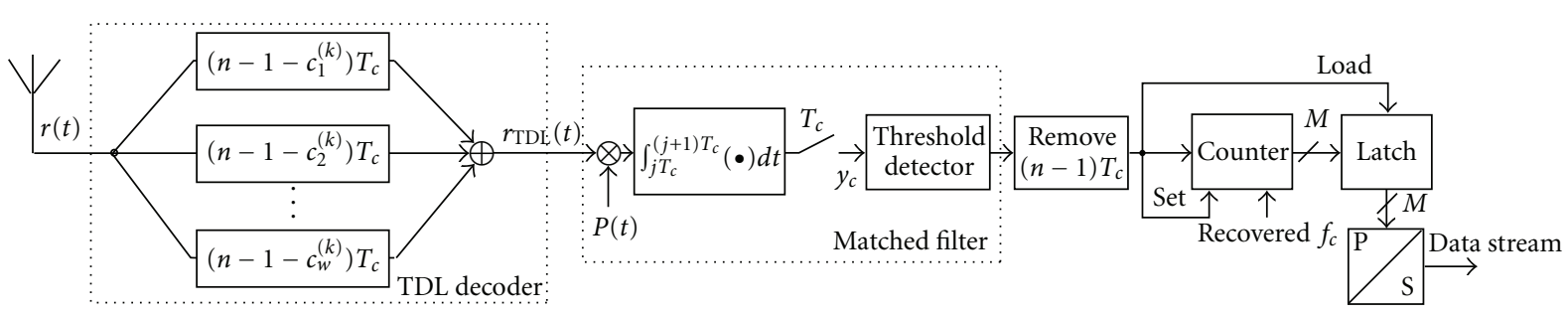

(b)

Figure 3: Proposed (a) transmitter and (b) receiver.

complexity. Figure 3 shows a TDL-based transmitter and receiver.

At the transmitter input, a serial data stream is transformed into a parallel $M$-bit data sequence and then loaded in the latch. Simultaneously with loading, the counter is reset and it starts to count with chip clock frequency. The outputs of the counter and latch are compared, and when outputs match, the comparator goes high which implies starting of the new symbol generation. Then, the new $M$-bit data sequence is loaded into the latch and the counter is reset/started. The comparator drives the pulse generator which generates a Gaussian monocycle when its input goes high and extra $(n-1)$ redundant empty chips are added. After that, the PIM sequence is fed into the TDL encoder. TDL consists of $w$ parallel tap-delay elements, where each element delay is determined by the codeword of the signature sequence. The signature sequence is obtained using an SOOC [15] defined by $\left(n, w, \lambda_{a}, \lambda_{c}\right)$, where $n$ is code length, $w$ is code weight, and $\lambda_{a}$ and $\lambda_{c}$ are unity auto- and cross-correlation constraints, respectively. The overall transmitted signal of the $k$ th user is given as follows:

$$
s^{(k)}(t)=\sum_{m=0}^{\infty} \sum_{j=1}^{w} \sqrt{E_{g}} p\left(t-c_{j}^{(k)} T_{c}-\left(n m+\sum_{l=-1}^{m-1} S_{l}^{(k)}\right) T_{c}\right),
$$

where $c_{j}^{(k)}$ is an element of the SOOC codeword and $S_{l}^{(k)}$ is the $k$ th user data sequence representing data coded into PIM. Due to the added redundant chips, the overall achievable data rate is decreased, and from (2) it can be written as follows:

$$
R_{b \text {-PIM }}=\frac{\log _{2} L}{\left(n+L_{\text {avg }}\right) T_{c}} .
$$

If AWGN is the only source of interference in the channel, the signal received in the multi-user environment is given as follows:

$$
r(t)=\sum_{k=1}^{N_{u}} s^{(k)}\left(t-\tau^{(k)}\right)+n(t),
$$

where $N_{u}$ is the number of users, $\tau^{(k)}$ is time delay of the $k$ th user (assumed to be the integer multiple of $T_{c}$ ), and $n(t)$ is an AWGN component with zero mean and variance $N_{0} / 2$.

At the input of the receiver, $r(t)$ is passed through the TDL decoder, where by tuning the delay elements a higher amplitude pulse can be formed by each of the pulses in the signature sequences. The decoded signal is then fed into the correlator-based matched filter which multiplies the signal by the template waveform. The decision which chip is empty and which chip contains a pulse is made on the basis of autocorrelation properties of the Gaussian monocycle at the threshold detector. At the output of the threshold detector, the transmitted PIM data encoded stream is estimated by removing redundant $(n-1)$ chips. The pulse at the start of the PIM symbol is then used to load the output of the counter in the latches and to reset the counter.

If we assume that the first user is the desired user, then the decoded signal at the input of the matched filter (after the TDL decoder) is given as follows:

$$
r_{\mathrm{TDL}}(t)=S^{(1)}+I_{\mathrm{SELF}}+I_{\mathrm{MUI}}+N,
$$




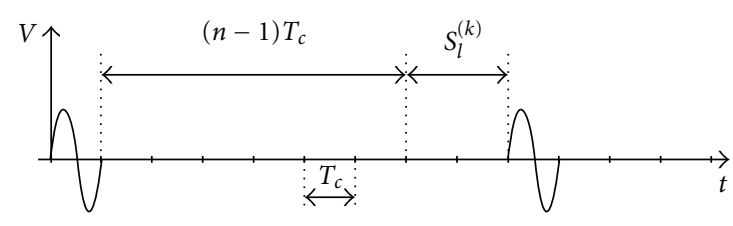

(a)

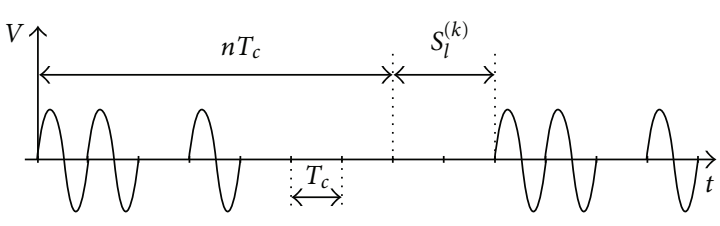

(b)

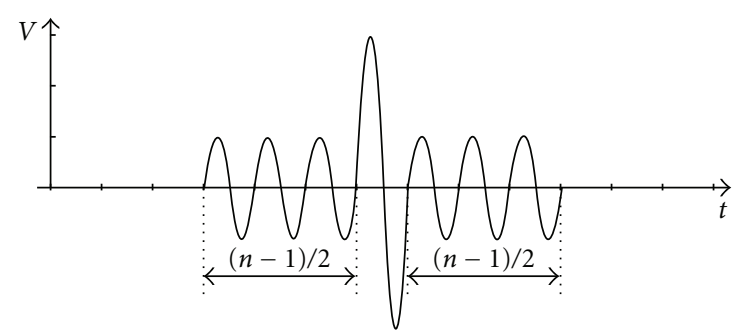

(c)

FIgURe 4: PIM symbol (a) before the TDL encoder, (b) after the TDL encoder, (c) after the TDL decoder.

where $S^{(1)}, I_{\mathrm{SELF}}, I_{\mathrm{MUI}}$, and $N$ are the desired PIM sequence, self-interference, interference due to the multiuser interference (MUI), and AWGN interference, respectively, given as follows:

$$
\begin{aligned}
S^{(1)}= & \sum_{m=0}^{\infty} w \sqrt{E_{g}} p \\
& \times\left(t-(n-1) T_{c}-\left(n m+\sum_{l=-1}^{m-1} S_{l}^{(1)}\right) T_{c}-\tau^{(1)}\right) \\
I_{\mathrm{SELF}}= & \sum_{m=0}^{\infty} \sum_{j=1}^{w} \sum_{J=1}^{w} \\
& \times \sqrt{E_{g}} p\left(t-c_{J}^{(1)} T_{c}-\left(n-1-c_{j}^{(1)}\right) T_{c}\right. \\
I_{\mathrm{MUI}}= & \left.\left.\sum_{m=0}^{\infty} \sum_{j=1}^{w} \sum_{j^{\prime}=1}^{w} \sum_{k=1}^{N_{u}-1} \sum_{l=-1}^{m-1} S_{l}^{(1)}\right) T_{c}-\tau^{(1)}\right) \\
& \times \sqrt{E_{g} p}\left(t-c_{j^{\prime}}^{(k)} T_{c}-\left(n-1-c_{j}^{(1)}\right) T_{c}\right. \\
N= & \sum_{j=1}^{w} n(t) . \\
& \left.-\left(n m+\sum_{l=-1}^{m-1} S_{l}^{(k)}\right) T_{c}-\tau^{(k)}\right)
\end{aligned}
$$

Figure 4 shows an example of the PIM symbol with the $(7,3,1,1)$ SOOC signature sequence.

\section{Error Performance Analysis}

In order to derive the error probability, some simplification has been assumed.

(a) Channel model is an AWGN channel (no multipath components).

(b) MUI is approximated as a Gaussian random variable.

(c) There is a perfect synchronization and power control between the transmitter and the receiver.

In order to recover the desired signal, at the input of the correlator-based matched filter (MF) the received signal is multiplied by the template signal $p(t)$ and then integrated over the chip time $T_{c}$ and a decision is made upon autocorrelation properties given as follows:

$$
\rho(\Delta t)=\int_{0}^{T_{c}} p(t) p(t-\Delta t) d t
$$

Note that

$$
\rho(\Delta t)= \begin{cases}1, & \Delta t=0, \\ 0, & \Delta t \geq\left|T_{c}\right| .\end{cases}
$$

The decision whether a pulse occurs in the current chip is made according to the threshold level $v_{t h} \sqrt{E_{g}}$ at the detector. When the pulse is detected, the data is recovered by removing redundant $(n-1)$ chips (due to the SOOC-CDMA) and counting empty chips employing the PIM demodulator. The error can occur in two ways. First, if the correct pulse is not detected, it would merge the previous and the current symbol. Second, if the false pulse is detected within empty $S_{l}^{(1)}$ chips, it would divide the current and the next symbol. In order to obtain the error probability, we will first derive the probability that a correct pulse is not detected. From Figure 4 , it can be seen that self-interference $I_{\text {SELF }}$ does not affect the chip where a correct pulse occurs, so the only inter- 
ference is due to the MUI and AWGN. The decision variable for the chip where the pulse occurs in the $m$ th symbol is

$$
y_{c}=w \sqrt{E_{g}} \rho(0)+I_{\text {MUI } \_m}+N,
$$

where $N$ is the AWGN interference, that is, a Gaussian random variable with zero mean and variance $w N_{0} / 2$ and $I_{\text {MUI } \_} m$ is the MUI in the $m$ th symbol given as

$$
I_{\text {MUI_m }}=\sum_{j=1}^{w} \sum_{j^{\prime}=1}^{w} \sum_{k=1}^{N_{u}-1} \sqrt{E_{g}} \rho\left(\delta_{\mathrm{MUI}}\right) .
$$

From [16] it can be seen that the probability that one pulse occurs in a single chip due to the MUI has a binomial distribution, so $\delta_{\mathrm{MUI}}$ can be modeled as a binomial random variable which can be either zero (meaning that the MUI pulse occurs in the current chip) or $\geq\left|T_{c}\right|$ (means that the MUI pulse does not occur in the current chip) with probabilities $P_{\mathrm{MUI}}$ and $\left(1-P_{\mathrm{MUI}}\right)$, respectively. The probability of one pulse interference due to the MUI is given from [16] as follows:

$$
P_{\mathrm{MUI}}=\frac{2}{2 n+2^{M}-1}
$$

where code length $n=N_{u} w(w-1)$ and $M$ is the number of bits per symbol. Probability density function (PDF) of $\delta_{\text {MUI }}$ is

$$
\operatorname{PDF}_{\mathrm{MUI}}(x)=\left(1-P_{\mathrm{MUI}}\right) \delta(x)+P_{\mathrm{MUI}} \delta(x-1) .
$$

By using the central limit theorem, $I_{\text {MUI } m}$ can be modeled as a Gaussian random variable with mean

$$
\begin{aligned}
E\left[I_{\text {MUI } \_m}\right] & =\sum_{j=1}^{w} \sum_{j^{\prime}=1}^{w} \sum_{k=1}^{N_{u}-1} E\left[\sqrt{E_{g}} \rho\left(\delta_{\mathrm{MUI}}\right)\right] \\
& =\left(N_{u}-1\right) w^{2} \sqrt{E_{g}} P_{\mathrm{MUI}},
\end{aligned}
$$

where $E\left[{ }^{*}\right]$ is the mean value operator. Variance of $I_{\text {MUI } m}$ is

$$
\begin{aligned}
\operatorname{Var}\left[I_{\text {MUI } \_m}\right]= & E\left[I_{\text {MUI } \_m}^{2}\right]-E\left[I_{\text {MUI } \_m}\right]^{2} \\
= & \sum_{j=1}^{w} \sum_{j^{\prime}=1}^{w} \sum_{k=1}^{N_{u}-1} E\left[\left(\sqrt{E_{g}} \rho\left(\delta_{\text {MUI }}\right)\right)^{2}\right] \\
& -\sum_{j=1}^{w} \sum_{j^{\prime}=1}^{w} \sum_{k=1}^{N_{u}-1} E\left[\sqrt{E_{g}} \rho\left(\delta_{\text {MUI }}\right)\right]^{2} \\
= & \left(N_{u}-1\right) w^{2} E_{g} P_{\text {MUI }}\left(1-P_{\text {MUI }}\right) .
\end{aligned}
$$

The decision variable $y_{c}$ can then be modeled as a Gaussian random variable with mean $w \sqrt{E_{g}}+E\left[I_{\text {MUI_m }}\right]$ and variance $\operatorname{Var}\left[I_{\text {MUI_m }}\right]+w N_{0} / 2$.

If $P_{p}$ denotes the probability of an error decision whether or not the pulse is detected in the current chip, then the probability that the correct pulse will not be detected is from [17] the probability equal to $P\left(y_{c}<v_{t h} \sqrt{E_{g}}\right)$ or

$$
P_{p}=Q\left(\sqrt{\frac{\left(w+\left(N_{u}-1\right) w^{2} P_{\mathrm{MUI}}-v_{t h}\right)^{2} E_{g}}{\left(\operatorname{Var}\left[I_{\text {MUI_m }}\right]+w N_{0} / 2\right)}}\right),
$$

where Q-function is defined as follows:

$$
Q(x)=\frac{1}{\sqrt{2 \pi}} \int_{x}^{\infty} e^{-t^{2} / 2} d t, \quad x \geq 0 .
$$

The second way that an error can occur is the probability that a false pulse is detected within an empty chip. The decision variable for the chip where the pulse does not occur in the $m$ th symbol is

$$
y_{c}=I_{\text {SELF } \_m}+I_{\text {MUL } \_m}+N,
$$

where $I_{\text {SELF_ } m}$ is self-interference due to $(w-1)$ uncorrelated pulses at the end of the TDL decoder in the $m$ th symbol. The probability of one pulse interference in a single chip due to self-interference also has a binomial distribution and it is given from [16] as follows:

$$
P_{\text {SELF }}=\frac{2}{2 n+2^{M}-3} .
$$

Using the same procedure for modeling $I_{\text {MUI } m}, I_{\text {SELF_ } m}$ can be modeled as a Gaussian random variable with mean

$$
E\left[I_{\text {SELF }-m}\right]=w(w-1) \sqrt{E_{g}} P_{\text {SELF }}
$$

and variance

$$
\operatorname{Var}\left[I_{\text {SELF } \_m}\right]=w(w-1) E_{g} P_{\text {SELF }}\left(1-P_{\text {SELF }}\right) .
$$

The decision variable $y_{c}$ can then be modeled as a Gaussian random variable with mean $E\left[I_{\text {MUI_m }}\right]+E\left[I_{\text {SELF_m }}\right]$ and variance $\operatorname{Var}\left[I_{\text {SELF } \_m}\right]+\operatorname{Var}\left[I_{\text {MUI } \_m}\right]+w N_{0} / 2$. If $P_{z}$ denotes the probability whether or not a pulse is detected in a wrong chip, then the probability that a wrong pulse will be detected is from [17] the probability equal to $P\left(y_{c}>v_{t h} \sqrt{E_{g}}\right)$ or

$P_{z}=Q\left(\sqrt{\frac{\left(v_{t h}-\left(w(w-1) P_{\text {SELF }}+\left(N_{u}-1\right) w^{2} P_{\text {MUI }}\right)\right)^{2} E_{g}}{\left(\operatorname{Var}\left[I_{\text {SELF_ }-m}\right]+\operatorname{Var}\left[I_{\text {MUI_m }}\right]+w N_{0} / 2\right)}}\right)$.

In order to compare the performance of PIM with other modulation techniques, a packet error rate (PER) is introduced [18]. A packet error occurs if one or more symbols within a packet are erroneous. If the packet containing $B$ data bits is considered, then the number of symbols and hence the number of transmitted pulses in a packet is $B / M$. Assuming that there is no guard slot in the symbol, the average number of empty slots per packet is $B\left(2^{M}-1\right) /(2 M)$. Therefore, the probability of the packet error is given by

$$
P_{\mathrm{PE}}=1-\left(1-P_{p}\right)^{B / M}\left(1-P_{z}\right)^{B\left(2^{M}-1\right) /(2 M)} .
$$

With (18), (24), and (25) the error probability is given as the function of energy per symbol, but in digital communication systems energy per bit $\left(E_{b}\right)$ is a natural figure of merit, so by replacing $E_{g}=M E_{b}$, we can obtain PER as a function of $E_{b} / N_{0}$. If we want to compare PER performance as the function of average signal power to the average noise power ratio (SNR), the following equation holds [19]:

$$
\frac{E_{b}}{N_{0}}=\operatorname{SNR} \frac{W}{R_{b}},
$$

where $W=1 / T_{c}$ is the channel bandwidth and $R_{b}$ is the bit rate defined in (2), (3). 


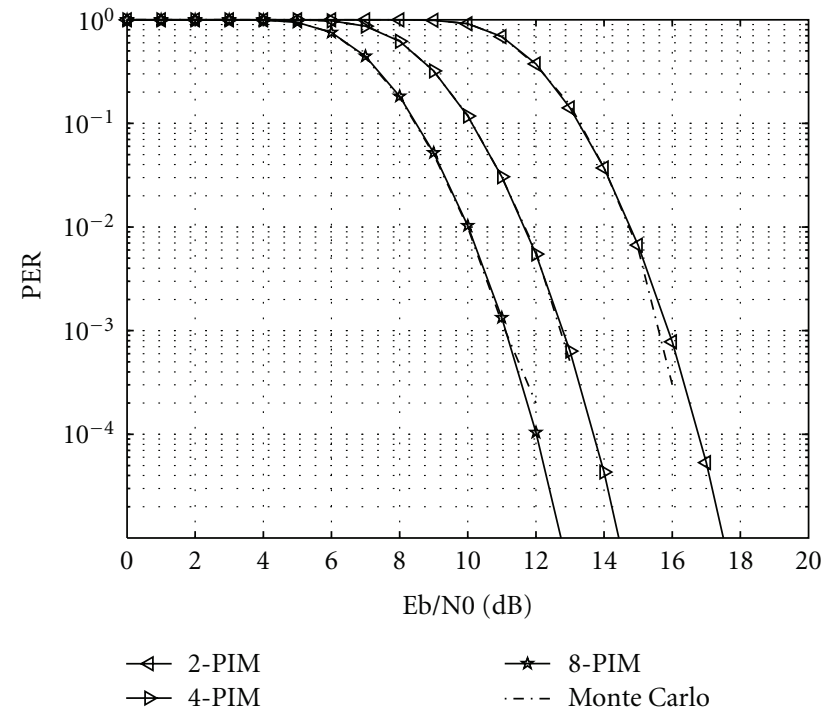

FIGURE 5: Comparison of PER performance between Monte Carlo simulation and the derived error probability for $B=128$.

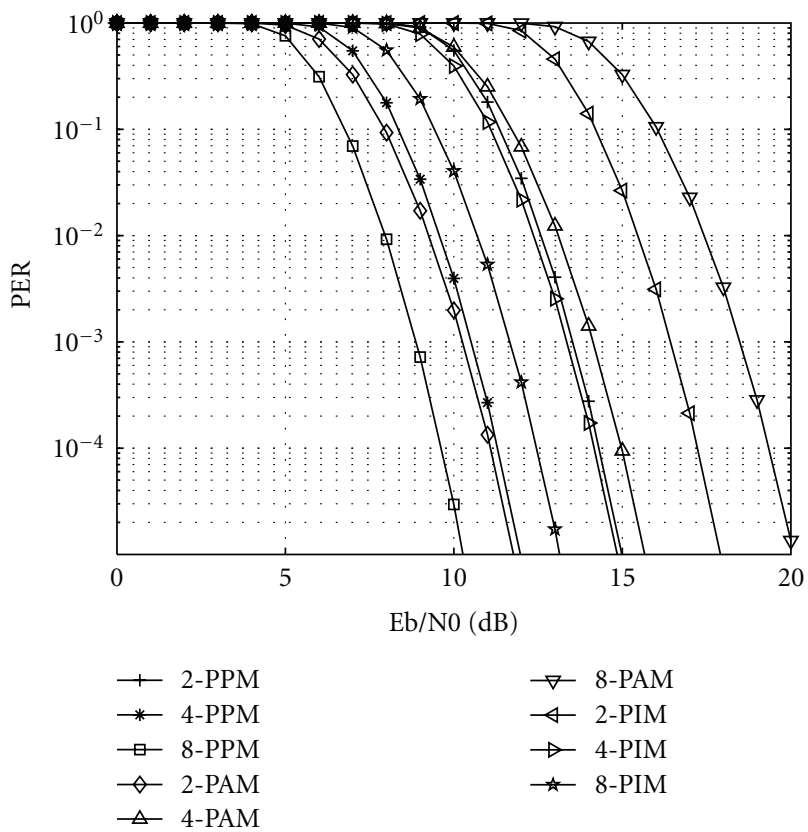

FIGURE 6: PER performance comparison of PAM, PPM, and PIM for the same bit energy.

\section{Simulation Results}

The error probability given by (18), (24), and (25) is compared with Monte Carlo simulation [20] in Figure 5 for three different PIMs and the packet length $B=128$. It can be seen that the derived error probability matches simulation results for 4-PIM and 8-PIM, while for 2-PIM there is a slight difference for large $E_{b} / N_{0}$.

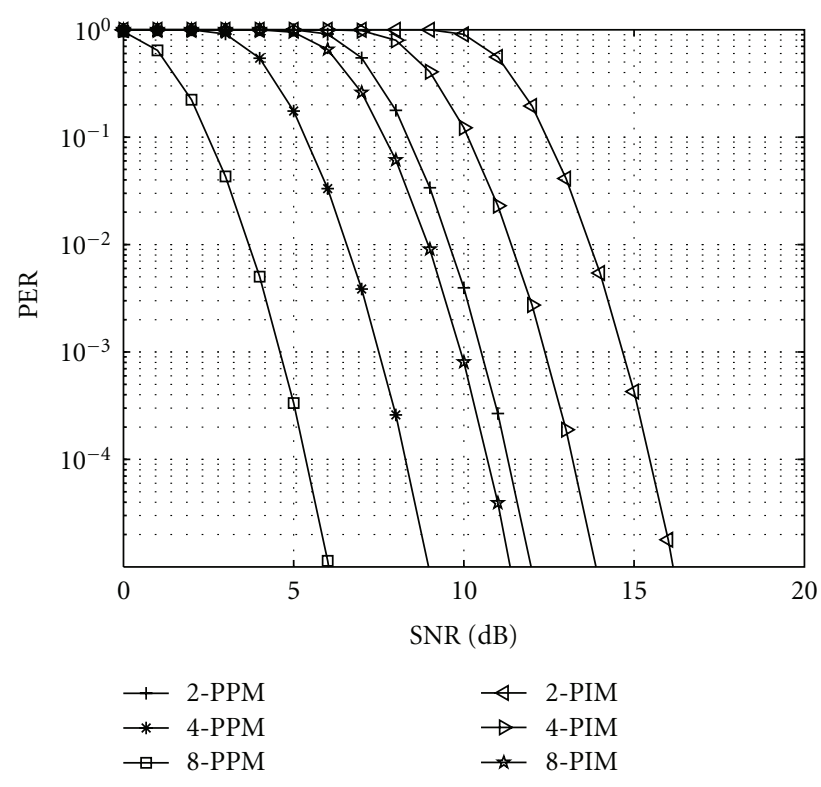

FIGURE 7: PER performance comparison of PPM and PIM for the same average power per symbol.

In order to compare PIM with PPM and PAM, packet length is chosen to be $B=512$ bits, $w=1$, and $v_{t h}=0.5$. PER performance of PIM is obtained using (18), (24), (25) and the results are shown in Figure 6. In the simulation, the number of modulation levels is $L=2,4,8$. In the case of $L=2$, PIM has a $6 \mathrm{~dB}$ and $3 \mathrm{~dB}$ worse performance than 2-PAM and 2-PPM, respectively. With the increase of the modulation level to $L=4$, PIM has a $0.8 \mathrm{~dB}$ better performance than 4 -PAM and $3 \mathrm{~dB}$ worse than 4 -PPM, while for $L=8$ PIM performance is $7 \mathrm{~dB}$ better than 8 -PAM and $3 \mathrm{~dB}$ worse than 8-PPM. Generally, it can be seen that if the modulation level increases, PIM and PPM performance increases while PAM decreases significantly.

To compare PIM with PPM for the same average power per symbol, equations (18), (24), (25) and (26) are used. Packet length is chosen to be $B=512$ bits, $w=1$ and $v_{t h}=0.5$. Results are shown in Figure 7. In the simulation the number of modulation levels is $L=2,4,8$. In the case of $L=2$, PIM has a $4 \mathrm{~dB}$ lower PER than 2-PPM. With the increase of the modulation level to $L=4$, 8 PIM performance decreases compared with PPM.

Figure 8 shows the influence of the threshold level $v_{t h}$ on the PER performance for 8-PIM when the code weight is $w$ $=10$. It can be seen that the optimal threshold is at $v_{t h}=$ 7. It results from the fact that in an 8-PIM symbol there is only one chip where a pulse occurs and on average 4.5 empty chips, so the probability that a false pulse will be detected is higher than the probability that a correct pulse will not be detected.

Figure 9 shows the influence of the code weight $w$ on PER performance for 8-PIM with $v_{t h}$ set to an optimal value. It can be seen that 8-PIM with $w=11$ has a slightly better performance than 8-PIM with $w=10$, and $2.3 \mathrm{~dB}$ better than 8-PIM with $w=9$. 


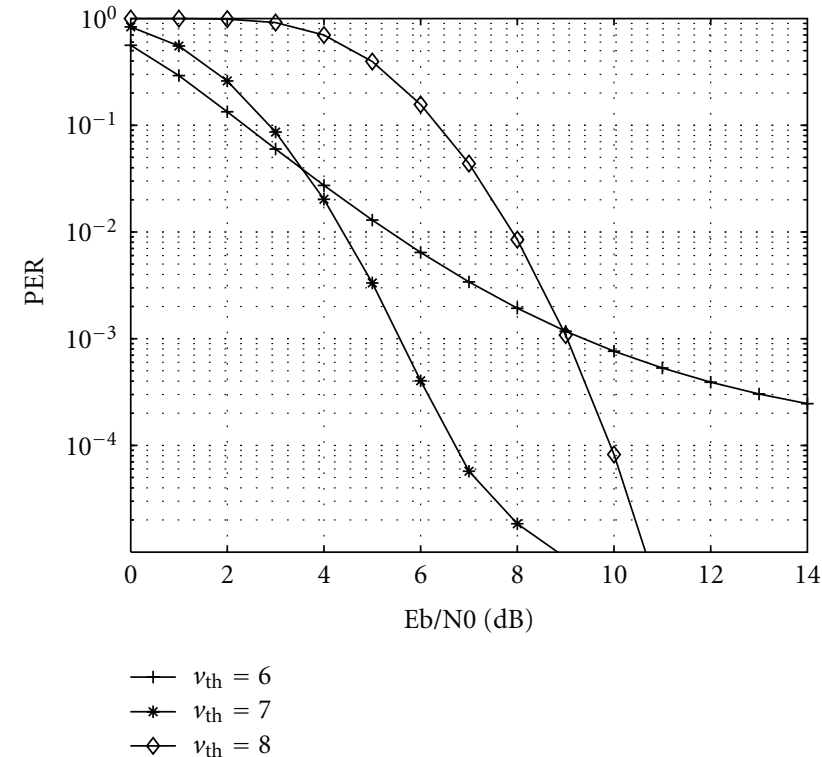

FIGURE 8: Influence of the $v_{t h}$ level on 8-PIM performance with code weight $w=10$.

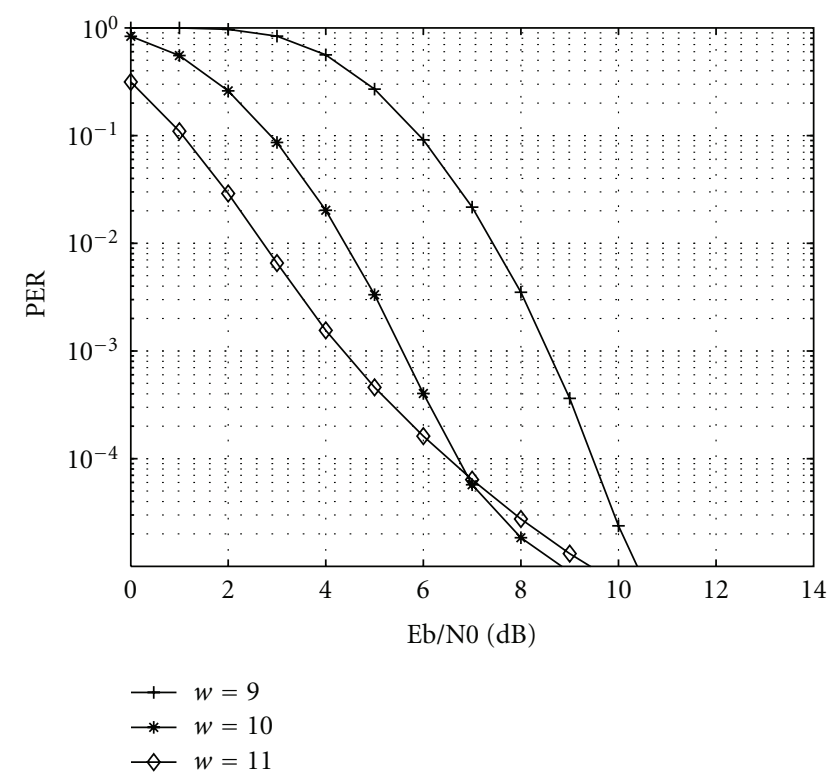

FIGURE 9: Influence of code weight $w$ on 8-PIM performance for the optimal $v_{t h}=7$.

Figure 10 shows the influence of the threshold level $v_{t h}$ on PER performance in the presence of the MUI for 8-PIM when the code weight is $w=10$ and $E_{b} / N_{0}=15 \mathrm{~dB}$. It can be seen that for the optimal threshold $v_{t h}=6$, the PER performance improves significantly.

Code weight $w$ influence on 8-PIM in presence of the MUI for optimal $v_{t h}$ is analyzed and shown in Figure 11. It can be seen that with an increase of code weight, PER is improved, which is a result of more correlated pulses at the receiver. This advantage is at the cost of the data rate shown from (6) in Figure 11.

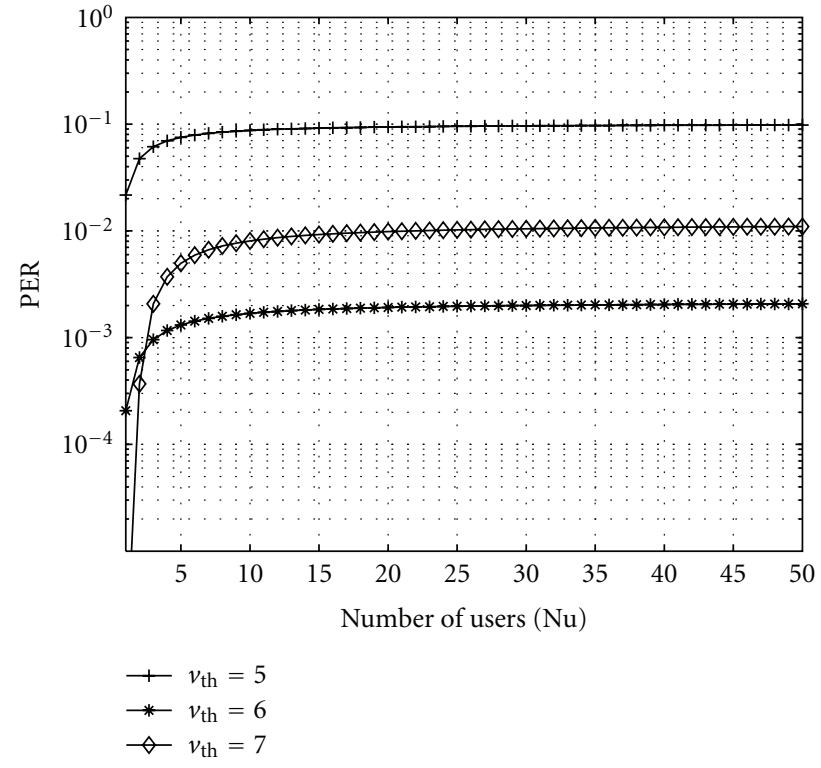

FIgURE 10: Influence of $v_{t h}$ on 8-PIM PER performance when the number of users increases, for $w=10$ and $E_{b} / N_{0}=15 \mathrm{~dB}$.

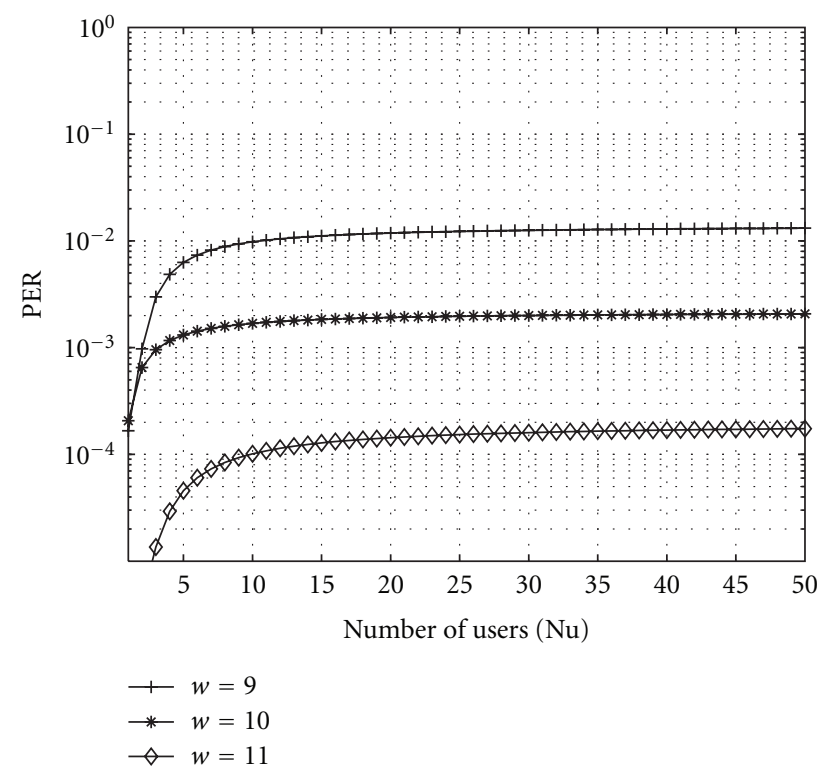

FIGURE 11: Influence of the number of users on 8-PIM performance for optimal $v_{t h}$ and $E_{b} / N_{0}=15 \mathrm{~dB}$.

\section{Conclusion}

This paper proposes an anisochronous PIM scheme for IRUWB communication systems. The basic principles and characteristics of anisochronous PIM scheme are outlined. Unlike PPM, PIM requires no symbol synchronization, which results in a much simpler receiver structure (only one correlator). The proposed multiple access method based on SOOC-TDL-CDMA allows a totally asynchronous transmission and it needs only chip synchronization which significantly reduces hardware complexity, while classical 


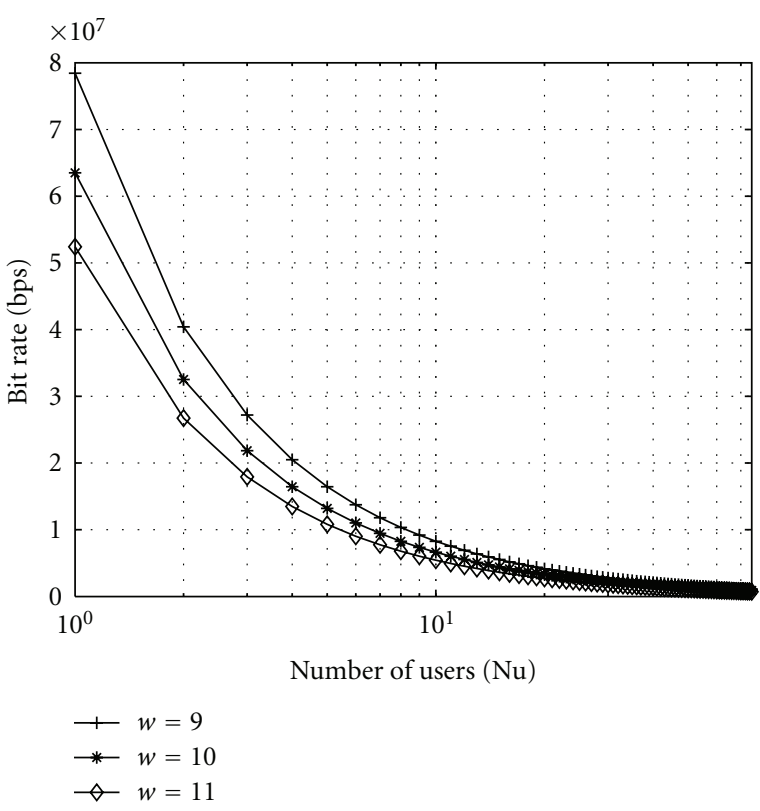

FIGURE 12: Influence of the number of users on the bit rate for 8PIM and $T_{c}=1 \mathrm{~ns}$.

time-hopping IR-UWB needs both frame and chip synchronization which increase hardware complexity. It is shown that an increase of code weight $w$ can decrease PER at the cost of hardware complexity (more delay elements at TDL) and the influence of $v_{t h}$ in both single- and multiuser environment is analyzed. The major disadvantage of anisochronous PIM techniques is that they have a variable symbol length, and hence the time required to transmit a data packet containing a fixed number of bits is not constant. Employing some form of a source coding scheme, packet length variation can be limited still maintaining the increase in information capacity over isochronous modulation techniques. Simpler receiver complexity and very high achievable bit-rates make PIM modulation very attractive for IR-UWB short-range communication systems.

\section{References}

[1] R. A. Scholtz, "Multiple-access performance limits with time hopping modulation," in Proceedings of the IEEE Military Communications Conference, pp. 11-14, October 1993.

[2] FCC, "Revision of part 15 of the commission's rules regarding ultra-wideband transsmision systems," Federal Communications Commission, ET Docket, pp. 98-153, 2002.

[3] Y. Kim, B. Jang, C. Shin, and B. F. Womack, "Orthonormal pulses for high data rate communications in indoor UWB systems," IEEE Communications Letters, vol. 9, no. 5, pp. 405$407,2005$.

[4] J. A. N. da Silva and M. L. R. de Campos, "Method for obtaining spectrally efficient orthogonal UWB pulse shapes," in Proceedings of the International Telecommunications Symposium (ITS '06), pp. 46-51, Fortaleza-CE, Brazil, September 2006.
[5] M. Ghavami, L. B. Michael, and R. Kohno, Ultra Wideband Signals and Systems in Communication Engineering, John Wiley \& Sons, New York, NY, USA, 2004.

[6] H. Zhang, W. Li, and T. A. Gulliver, "Pulse position amplitude modulation for time-hopping multiple-access UWB communications," IEEE Transactions on Communications, vol. 53, no. 8, pp. 1269-1273, 2005.

[7] H. Zhang and T. A. Gulliver, "Biorthogonal pulse position modulation for time-hopping multiple access UWB communications," IEEE Transactions on Wireless Communications, vol. 4, no. 3, pp. 1154-1162, 2005.

[8] S. Majhi and A. S. Madhukumar, "Combining OOK with PSM modulation for TH-UWB radio systems: a performance analysis," EURASIP Journal on Wireless Communications and Networking, vol. 2008, 2008.

[9] C. J. Mitchell, G. T. F. de Abreu, and R. Kohno, "Combined pulse shape and pulse position modulation for high data rate transmissions in ultra-wideband communications," International Journal of Wireless Information Networks, vol. 10, no. 4, pp. 167-178, 2003.

[10] M. Herceg, T. Matic, and T. Svedek, "Performances of hybrid amplitude shape modulation for UWB communications systems over AWGN channel in the single and multi-user environment," Radioengineering Journal, vol. 18, no. 3, 2009.

[11] M. Herceg, T. Matic, and T. Svedek, "Performance of hybrid pulse shape amplitude modulation for UWB communications systems over multipath channels," in Proceedings of the 9th International Conference on Telecommunication in Modern Satellite, Cable, and Broadcasting Services (TELSIKS '09), October 2009.

[12] Z. Ghassemlooy, A. R. Hayes, N. L. Seed, and E. D. Kaluarachchi, "Digital pulse interval modulation for optical communications," IEEE Communications Magazine, vol. 36, no. 12, pp. 95-99, 1998.

[13] T. Svedek and S. Rupcic, "Anisochronous modulation methods for optical short-range wireless comunnications," in Proceedings of IEEE International Conference on Intelligent Engineering Systems, L. Žlajpah and I. J. Rudas, Eds., 2000.

[14] C. K. See, Z. Ghassemlooy, and J. M. Holding, "Hybrid PIMCDMA for optical wireless networks," in Proceedings of the 1st Annual PostGraduate Symposium on the Convergence of Telecommunications, Networking and Broadcasting (PGNET '00), pp. 195-200, June 2000.

[15] J.-G. Zhang, "Strict optical orthogonal codes for purely asynchronous code-division multiple-access applications," Applied Optics, vol. 35, no. 35, pp. 6996-6999, 1996.

[16] Z. Ghassemlooy and C. K. See, "Symbol and bit error rates analysis of hybrid PIM-CDMA," EURASIP Journal on Wireless Communications and Networking, vol. 2005, Article ID 162417, 8 pages, 2005.

[17] J. G. Proakis, Digital Communications, McGraw-Hill, New York, NY, USA, 4th edition, 2001.

[18] Z. Ghassemlooy and A. R. Hayes, "Digital pulse interval modulation for IR communication systems-a review," International Journal of Communication Systems, vol. 13, no. 7-8, pp. 519-536, 2000.

[19] B. Sklar, Digital Communications: Fundamentals and Applications, Prentice-Hall, New York, NY, USA, 2nd edition, 2001.

[20] J. G. Proakis and M. Salehi, Contemporary Communication Systems Using MATLAB, PWS, Boston, Mass, USA, 1998. 